\title{
OPEN Diurnal preference and depressive symptomatology: a meta-analysis
}

\author{
Ray Norbury
}

Eveningness, a preference for later sleep and rise times, has been associated with a number of negative outcomes in terms of both physical and mental health. A large body of evidence links eveningness to Major Depressive Disorder (MDD). However, to date, evidence quantifying this association is limited. The current meta-analysis included 43 effect sizes from a total 27,996 participants. Using a random-effects model it was demonstrated that eveningness is associated with a small effect size (Fisher's $Z=-2.4,95 \% \mathrm{Cl}[-0.27 .-0.21], p<0.001$ ). Substantial heterogeneity between studies was observed, with meta-regression analyses demonstrating a significant effect of mean age on the association between diurnal preference and depression. There was also evidence of potential publication bias as assessed by visual inspection of funnel plots and Egger's test. The association between diurnal preference and depression is small in magnitude and heterogenous. A better understanding of the mechanistic underpinnings linking diurnal preference to depression and suitably powered prospective studies that allow causal inference are required.

Circadian rhythms are endogenous processes that follow a near 24-h cycle. These rhythms are self-autonomous, and in humans are controlled by a central oscillator (or master clock) located in the suprachiasmatic nuclei (SCN) of the hypothalamus ${ }^{1}$. Individuals maintained under constant conditions isolated from external timing cues can maintain an endogenous period close to $24 \mathrm{~h}$. However, because these cycles oscillate with periods that differ slightly from $24 \mathrm{~h}$ there is a loss of synchrony with the earth's day-night cycle. To account for this, the central oscillator is adaptable and can be entrained to respond to external time-givers, or zeitgebers (e.g., light). The molecular mechanisms underlying the generation of circadian oscillations relies on multiple proteins generated by clock-related genes that interact to inhibit and activate gene expression in an inhibitory loop that oscillates to produce the near-24-h cycle.

Disruption of circadian rhythms are widely reported in depression. Patients may display a regular daily pattern of symptoms with increased symptom severity often reported in the morning ${ }^{2}$. In MDD there are dampened and phase-shifted rhythms of activity, temperature and hormones (with the exception of cortisol which is increased rather than reduced $)^{3}$ which are correlated with depressive symptom severity ${ }^{4}$. Disrupting circadian rhythms through shift-work, long-distance travel (jet-lag) or misalignment between internal (biological) and social (external) time (referred to as social jet-lag) has been observed to increase depressive symptoms ${ }^{5-7}$. Poor entrainment of the SCN to light (as may occur in Major Depressive Disorder with Seasonal Pattern) and the subsequent disruptions of daily rhythms of hormones and neurotransmitters has been reported as an additional factor to develop depression ${ }^{8}$. In addition, treatments that target circadian rhythms (e.g. agomelatine, early morning bright-light therapy) may be effective in reducing depressive symptoms ${ }^{9}$.

Diurnal preference, or morningness/eveningness, reflects an individual's preferred timings for sleep and activity and is an individual trait that arises through a combination of endogenous factors and external, environmental stimuli $^{10}$. Evening-types (colloquially referred to as "night owls") prefer to go to bed late, rise late and plan work and other activities (e.g., meetings, gym visits) for later in the day. By contrast, morning-types ("larks") prefer to retire early, rise early and plan activities for earlier in the day.

Diurnal preference can be reliably estimated using subjective assessment, which has particular utility for large scale studies. A number of instruments have been developed to measure eveningness and among the most widely used are the Morningness-Eveningness Questionnaire (MEQ) ${ }^{11}$, the reduced version of the MEQ ${ }^{12}$ and the Composite Scale of Morningness $(\mathrm{CSM})^{13}$. All three measures have been shown to have moderate to good reliability and construct validity ${ }^{14}$ and the MEQ has also been demonstrated to be a strong predictor of dim melatonin onset-which is considered the most reliable measure of circadian rhythm in humans ${ }^{15}$.

Twin studies indicate heritability estimates between 46 and $57 \%$ for diurnal preference ${ }^{16}$ and diurnal preference is considered a relatively stable trait in adulthood ${ }^{17}$. A better understanding of individual differences in sleep-wake behaviour and how these may relate to disease is becoming increasingly important as a large body 
of work now suggests that eveningness is associated with a number of negative physical ${ }^{18}$ and mental health outcomes ${ }^{19,20}$ particularly depression ${ }^{21,22}$. Current evidence suggests that eveningness is associated with greater depressive symptomatology $y^{7,22,23}$. Eveningness is also associated with having a current diagnosis of depression, treatment for depression or antidepressant use $\mathrm{u}^{22,24,25}$, non-remission ${ }^{26}$ and suicidal thoughts ${ }^{27}$.

Despite the prevalence and debilitating nature of depression and the overwhelming body of evidence indicating an association between depression and diurnal preference ${ }^{8-17}$ synthesis of these data has been largely restricted to systematic reviews (e.g. ${ }^{19,20,28,29}$ ). To date, only one study has quantified this relationship in the form of a meta-analysis ${ }^{30}$. The aim of the current study was to extend this previous meta-analysis ${ }^{30}$ (which was limited to data published up to February 2016) to include recent literature published up to 31st December 2020. Based on previous work it is hypothesised that eveningness will be associated with greater depressive symptomatology.

\section{Methods}

The protocol for this meta-analysis was prospectively registered with PROSPERO (CRD4202122977). The raw data and fully reproducible code are available on the OSF (https://osf.io/wyjtx/).

Literature search. PubMed and Web of Science were searched for articles published between 1st January 2000 and 31st December 2020 using the search terms ("chronotype" OR "diurnal preference" OR "circadian preference" OR "morningness" OR "eveningness" OR "social jetlag") AND ("depression" OR "MDD" OR "Unipolar"). The titles and abstracts of articles returned using this search were initially screened before the full text was examined in greater detail.

To be included in the meta-analysis articles were required to meet the following criteria: (1) Diurnal preference quantified using either the Morning-Evening Questionnaire ${ }^{11}$ the Reduced Morningness-Eveningness Questionnaire $^{12}$ or the Composite Scale of Morningness ${ }^{13}$; (2) Depressive symptomatology measured using either the Beck Depression Inventory (BDI) ${ }^{31}$, the Hospital Anxiety and Depression Scale (HADS) ${ }^{32}$, the Hamilton Rating Scale for Depression (HRSD) ${ }^{33}$, the Depression, Anxiety, Stress Scales (DASS) ${ }^{34}$, the Quick Inventory of Depressive Symptomatology-Self-report (QUIDS-SR) ${ }^{35}$, the Centre for Epidemiology Studies Depression $(\mathrm{CES}-\mathrm{D})^{36}$, the Self-Rating Depression Scale (SDS $)^{37}$, the Montgomery-Asberg Depression Rating Scale-Self $(\text { MADRS-S })^{38}$, the Patient Health Questionnaire 9 (PHQ-9) $)^{39}$, the Patient Health Questionnaire 4 (PHQ-4) ${ }^{40}$, the Brief Symptom Rating Scale (BSRS ${ }^{41}$, the Geriatric Depression Scale (GDS) ${ }^{42}$ and the Depressed Mood Scale (DMS $)^{43}$; (3) Sufficient statistical information to estimate an effect size (correlation coefficient, mean and standard deviation or standard error, odds ratio); (4) Written in English; (5) Participants aged 18 or over; (6) For clinical samples, patients diagnosed with MDD; and (5) Published in a peer-reviewed journal. Exclusion criteria were: (1) Studies that included depressive disorders other than MDD (e.g., Seasonal Affective Disorder); and (2) Incomplete or modified versions of the MEQ/rMEQ/CSM (e.g. diurnal preference determined using a single question). Data selection is summarised in Fig. S1, Supplemental Data.

Study quality was evaluated using the McMaster critical review tool for quantitative studies ${ }^{44}$. Data extracted from the included studies was: (1) Authorship; (2) Year of publication; (3) Sample size; (4) Mean age; (5) Age range; (6) Measure of diurnal preference (e.g., MEQ); (7) Depression measure (e.g. BDI); (8) Gender breakdown (e.g. percentage of female participants); and (9) Clinical or non-clinical samples.

Statistical analyses. All statistical analyses were performed using R version $3.6 .1^{45}$ including the following packages: esc; effectsize; meta; metafor; dmetar: DiagrammeR; DiagrammeRsvg; and ggplot2. Individual effect sizes were obtained from each study. As most studies (52\%) reported correlation coefficients, Fisher's Z transformed correlation coefficient was used as the summary effect size. Odds ratios and standardised mean differences were transformed to Fisher's $\mathrm{Z}$ scores for inclusion in the analysis. The corresponding pooled effect size and its $95 \%$ confidence intervals (CI) were calculated from a random-effects model with a Sidik-Jonkman estimator for $\tau^{2}$ with Hartung-Knapp adjustment. This method was adopted as a conservative approach in the presence of sample heterogeneity ${ }^{46}$. A pooled effect size of $0.1-0.3$ was considered small, $0.3-0.5$ medium and $0.5-1$ considered a large effect ${ }^{47,48}$. Study heterogeneity was assessed with Q statistics and the $I^{2}$ index. Outlier analysis (studies were considered outliers if the $95 \%$ CI was outside the pooled effect size $95 \% \mathrm{CI}$ ) were also performed and the random-effects model refitted after excluding any such study. To assess the potential impact of publication bias, funnel plots were visually inspected, and an Egger's test conducted to quantify asymmetry with a $p$ value of $<0.05$ considered evidence of asymmetry that may reflect publication bias. Based on the outcome of the Egger's test, a trim and fill procedure was conducted to impute potential missing studies into the funnel plot to achieve symmetry. Categorial moderators (clinical vs. non-clinical, diurnal preference measure, clinical measure and published in $2020 v s$ any other year (this latter analysis was included as studies published in 2020 overlap with the COVID-19 outbreak and may therefore include data collected during the pandemic) were investigated uses subgroup analyses. Continuous variables (age, year of publication, sample size and percentage of female participants) were explored using meta-regression.

\section{Results}

The initial literature search returned 864 articles (PubMed $=318$, Web of Science $=546$, see Supplemental S1 for a graphical overview). Following article screening, a total of 51 studies were entered into the initial random effects model. Outlier analyses identified a total of ten studies ${ }^{49-58}$ and these were subsequently excluded from the analysis (See Supplemental S2 for details of these studies including the 95\% CI for each excluded effect size). Heterogeneity was reduced after excluding these studies (prior to exclusion $I^{2}=77.9 \%, \mathrm{Q}(52)=235.11, p<0.001$, after exclusion $I^{2}=60.2 \%, \mathrm{Q}(42)=105.5, p<0.001$ ) but remained substantial and significant. The final sample included 43 effect sizes from a total 27,996 participants with a mean age of 32 years. The predominant measure 


\begin{tabular}{|c|c|c|c|c|c|c|}
\hline Author & Chronotype & Age & $\mathbf{N}$ & Females (\%) & Sample & Depression measure \\
\hline Akram et al..$^{59}$ & MEQ & 24 & 453 & 75 & Non-clinical & HADS-depression subscale \\
\hline Asarnow et al. ${ }^{60}$ & CSM & 43 & 139 & 66 & Clinical & HRSD \\
\hline Aydin et al. ${ }^{61}$ & MEQ & 22 & 209 & 49 & Non-clinical & DASS-21 depression subscale \\
\hline Bakotic et al. ${ }^{62}$ & CSM & 21 & 1052 & 62 & Non-clinical & HADS-depression subscale \\
\hline Berdynaj et al. ${ }^{63}$ & MEQ & 21 & 86 & 78 & Non-clinical & DMS \\
\hline Chan et al. ${ }^{26}$ & MEQ & 51 & 253 & 84 & Clinical & BDI \\
\hline Coleman and Cain ${ }^{64}$ & rMEQ & 46 & 424 & 73 & Clinical & HRSD \\
\hline Furusawa et al. ${ }^{65}$ & MEQ & 41 & 362 & 0 & Non-clinical & SDS \\
\hline Gaspar-Barba et al. ${ }^{27}$ & MEQ & 32 & 82 & 37 & Clinical & BDI \\
\hline Haraszti et al. ${ }^{66}$ & MEQ & 38 & 44 & 100 & Non-clinical & PHQ-9 \\
\hline Hidalgo et al. ${ }^{23}$ & MEQ & 31 & 142 & 61 & Non-clinical & QUIDS-SR \\
\hline Hirata et al. ${ }^{67}$ & MEQ & 22 & 33 & 52 & Non-clinical & SDS \\
\hline Horne et al..$^{53}$ & rMEQ & 24 & 167 & 77 & Non-clinical & CES-D \\
\hline Hou et al. ${ }^{68}$ & MEQ & 37 & 884 & 63 & Non-clinical & CES-D \\
\hline Hsu et al. ${ }^{69}$ & MEQ & 20 & 790 & 53 & Non-clinical & MADRS \\
\hline Inomata et al. ${ }^{70}$ & MEQ & 21 & 27 & 48 & Non-clinical & CES-D \\
\hline Jankowski and Dmitrzak-Weglarz ${ }^{71}$ & CSM & 32 & 338 & 51 & Non-clinical & HADS-depression subscale \\
\hline Jankowski $^{72}$ & CSM & 22 & 974 & 70 & Non-clinical & PHQ-4-depression subscale \\
\hline Jeon et al. ${ }^{73}$ & MEQ & 32 & 700 & 91 & Non-clinical & CES-D \\
\hline Kang et al. ${ }^{74}$ & rMEQ & 19 & 940 & 63 & Non-clinical & DASS-21 depression subscale \\
\hline Khan et al. ${ }^{75}$ & MEQ & 40 & 59 & 54 & Non-clinical & BSRS \\
\hline Lau et al. ${ }^{76}$ & CSM & 21 & 230 & 66 & Non-clinical & CES-D \\
\hline Lester $^{77}$ & MEQ & 22 & 194 & 72 & Non-clinical & CES-D \\
\hline Liberman et al. $^{78}$ & MEQ & 19 & 242 & 64 & Non-clinical & CES-D \\
\hline Lin et al. ${ }^{79}$ & rMEQ & 27 & 1791 & 70 & Non-clinical & CES-D \\
\hline Markarian et al. ${ }^{80}$ & MEQ & 38 & 296 & 60 & Non-clinical & HADS-depression subscale \\
\hline Müller et al. ${ }^{81}$ & MEQ & 42 & 64 & 61 & Clinical & BDI \\
\hline Ong et al. ${ }^{82}$ & CSM & 49 & 156 & NR & Non-clinical & BDI-short form \\
\hline Park et al. ${ }^{83}$ & CSM & 19 & 5632 & 51 & Non-clinical & DASS-21 depression subscale \\
\hline Park $^{84}$ & CSM & 37 & 29 & 72 & Clinical & BDI \\
\hline Przepiorka et al.$^{85}$ & CSM & 20 & 398 & 71 & Non-clinical & BDI \\
\hline Randler et al. ${ }^{86}$ & CSM & 22 & 277 & 100 & Non-clinical & HADS-depression subscale \\
\hline Selvi et al..$^{5 *}$ & MEQ & 31 & 80 & 55 & Non-clinical & BDI-II \\
\hline Smagula et al. ${ }^{87}$ & CSM & 70 & 54 & 70 & Non-clinical & PHQ-9 \\
\hline Sun et al. ${ }^{88}$ & rMEQ & 48 & 629 & NR & Non-clinical & CES-D \\
\hline Togo et al. ${ }^{89}$ & MEQ & 41 & 2669 & 97 & Non-clinical & CES-D \\
\hline Toomey et al..$^{90}$ & MEQ & 55 & 1231 & NR & Non-clinical & BDI \\
\hline Türkoglu and Selvi ${ }^{91}$ & MEQ & 42 & 70 & 83 & Non-clinical & CES-D \\
\hline Üzer and Yücens ${ }^{58 *}$ & MEQ & 65 & 70 & 50 & Non-clinical & PHQ-9 \\
\hline Üzer and Yücens ${ }^{92}$ & MEQ & 22 & 339 & 54 & Non-clinical & BDI \\
\hline Watts and Norbury ${ }^{93}$ & rMEQ & 26 & 240 & 79 & Non-clinical & BDI \\
\hline Zhang et al. ${ }^{94}$ & MEQ & 19 & 616 & 65 & Non-clinical & PHQ-9 \\
\hline Zhou et al. ${ }^{95}$ & MEQ & 19 & 4531 & 30 & Non-clinical & HADS-depression subscale \\
\hline
\end{tabular}

Table 1. Study characteristics. For abbreviations please see main text. ${ }^{*}$ Two effect sizes were initially estimated for each of these studies two of which were subsequently identified outliers and excluded from the main random effects model.

of eveningness was the MEQ (59\% of included studies), CES-D and BDI where the most common measures of depressive symptomatology (respectively, 26\% and 19\%) and non-clinical samples made up $86 \%$ of effect sizes. Details of the included studies are shown in Table 1.

The effect size estimated from the random-effects model was Fisher's $Z=-0.24,95 \%$ CI [-0.27. -0.21 , $p<0.001$, demonstrating a small, but significant, association between eveningness and depression symptomatology (Fig. 1). Visual inspection of the funnel plot (Fig. 2) and the result of the Egger's test of the intercept $(-1.135,95 \%$ CI $[-1.86,-0.41], p=0.004)$ suggest publication bias should be taken into consideration when interpreting the results and the trim and fill adjusted estimate was $-0.21,95 \% \mathrm{CI}[-0.2438 ;-0.1715], p<0.0001$ with twelve adjusted studies. 
Author

Akram et al., 2019

Aydin et al., 2019

Bakotic et al., 2017

Berdynaj et al., 2016

Chan et al., 2014

Coleman \& Cain, 2019

Furusawa et al., 2015

Gaspar-Barba et al., 2009

Haraszti et al., 2014

Hidalgo et al., 2009

Hirata et al., 2007

Horne et al., 2018

Hou et al., 2020

Hsu et al., 2012

Inomata et al., 2014

Jankowski \& Dmitrzak-Weglarz, 2017

Jankowski, 2016

Jeon et al., 2017

Kang et al., 2020

Khan et al., 2020

Lester, 2015

Liberman et al., 2018

Lin et al., 2020

Markarian et al., 2019

Müller et al., 2016

Ong et al., 2007

Park et al., 2018

Park, 2020

Przepiorka et al., 2020

Randler et al., 2012

Selvi et al., 2010*

Smagula et al., 2020

Sun et al., 2020

Togo et al., 2017

Toomey, 2015

Türko?lu, 2020

Üzer \& Yücens, 2020b

Watts, 2017

Zhang, 2018

Zhou, 2020

Overall effect

Prediction interval

Heterogeneity: $p<0.01$
Asarnow et al., 2019

Lau et al., 2017

Üzer \& Yücens, 2020a

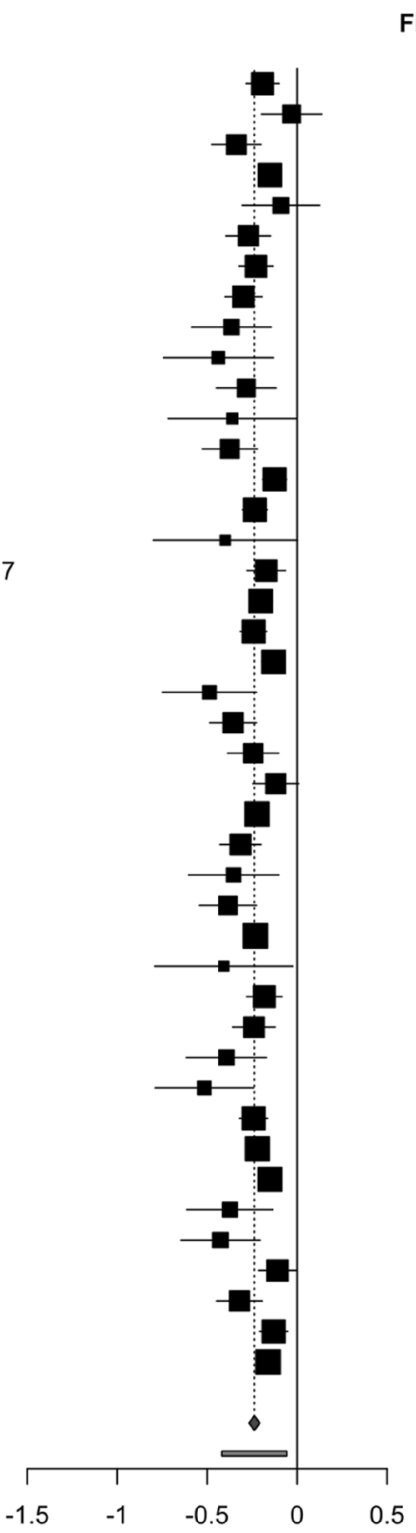

Fisher's Z 95\%-Cl Weight

$-0.19[-0.28 ;-0.10] \quad 2.9 \%$

$-0.03[-0.20 ; 0.14] \quad 1.9 \%$

$-0.34[-0.47 ;-0.20] \quad 2.3 \%$

$-0.15[-0.21 ;-0.09] \quad 3.3 \%$

$-0.09[-0.31 ; 0.12] \quad 1.5 \%$

$-0.27[-0.40 ;-0.15] \quad 2.4 \%$

$-0.23[-0.32 ;-0.13] \quad 2.8 \%$

$-0.30[-0.40 ;-0.19] \quad 2.7 \%$

$-0.37[-0.59 ;-0.14] \quad 1.4 \%$

$-0.44[-0.74 ;-0.13] \quad 0.9 \%$

$-0.28[-0.45 ;-0.12] \quad 1.9 \%$

$-0.36[-0.72 ; 0.00] \quad 0.7 \%$

$-0.37[-0.53 ;-0.22] \quad 2.1 \%$

$-0.12[-0.19 ;-0.06] \quad 3.2 \%$

$-0.23[-0.30 ;-0.16] \quad 3.2 \%$

$-0.40[-0.80 ; 0.00] \quad 0.6 \%$

$-0.17[-0.28 ;-0.06] \quad 2.7 \%$

$-0.20[-0.27 ;-0.14] \quad 3.3 \%$

$-0.24[-0.32 ;-0.17] \quad 3.1 \%$

$-0.13[-0.19 ;-0.07] \quad 3.3 \%$

$-0.49[-0.75 ;-0.23] \quad 1.1 \%$

$-0.36[-0.49 ;-0.23] \quad 2.4 \%$

$-0.24[-0.39 ;-0.10] \quad 2.2 \%$

$-0.12[-0.25 ; 0.01] \quad 2.4 \%$

$-0.22[-0.27 ;-0.18] \quad 3.5 \%$

$-0.32[-0.43 ;-0.20] \quad 2.6 \%$

$-0.35[-0.60 ;-0.10] \quad 1.2 \%$

$-0.38[-0.54 ;-0.23] \quad 2.0 \%$

$-0.23[-0.26 ;-0.21] \quad 3.6 \%$

$-0.41[-0.79 ;-0.02] \quad 0.6 \%$

$-0.18[-0.28 ;-0.08] \quad 2.8 \%$

$-0.24[-0.36 ;-0.12] \quad 2.5 \%$

$-0.39[-0.62 ;-0.17] \quad 1.4 \%$

$-0.52[-0.79 ;-0.24] \quad 1.1 \%$

$-0.24[-0.32 ;-0.16] \quad 3.1 \%$

$-0.22[-0.26 ;-0.18] \quad 3.5 \%$

$-0.15[-0.21 ;-0.10] \quad 3.4 \%$

$-0.37[-0.61 ;-0.13] \quad 1.3 \%$

$-0.43[-0.65 ;-0.21] \quad 1.4 \%$

$-0.11[-0.22 ; 0.00] \quad 2.7 \%$

$-0.32[-0.45 ;-0.19] \quad 2.4 \%$

$-0.13[-0.21 ;-0.05] \quad 3.1 \%$

$-0.16[-0.19 ;-0.13] \quad 3.6 \%$

$-0.24[-0.27 ;-0.21] 100.0 \%$

$[-0.42 ;-0.06]$

Figure 1. Forest plot of standardised effect sizes from each study. The overall effect is indicated in blue, prediction interval in red.

The substantial heterogeneity between studies suggests a potential impact of moderator variables on the reported association between eveningness and depressive symptomatology. To explore this a series of sub-group meta-analyses were conducted based on four potential confounders: Sample composition (clinical $v s$. nonclinical); Publication year (2020 vs. other year); Eveningness measure (MEQ vs. CSM vs. rMEQ) and Depression measure (BDI vs. CES-D vs. other). None of the included categorical moderators significantly explained heterogeneity in effect size. A summary of subgroup analyses is presented in Table 2. Meta-regression demonstrated that age was negatively associated with depression symptomatology $(\beta=-0.003, p=0.03,95 \% \mathrm{CI}[-0.005,-0.0003]$, $\mathrm{R}^{2}=10.63$, Fig. 3). Neither sample size $\left(\beta=0.00, p=0.12,95 \% \mathrm{CI}[-0.00,0.00], \mathrm{R}^{2}=4.44\right)$, year of publication $\left(\beta=-0.02, p=0.56,95 \%\right.$ CI $\left.[-0.09,0.05], \mathrm{R}^{2}=0\right)$ or percentage of female participants $(\beta=0.00, p=0.95,95 \%$ CI $\left.[-0.002,0.002], R^{2}=0\right)$ were related to the observed association between diurnal preference and depressive symptomatology.

\section{Discussion}

The current findings demonstrate a small but significant association between diurnal preference and depressive symptomatology. All of the reported studies indicated a positive association between eveningness and depression, ranging between -0.52 and -0.03 . The summary effect size for the random effects model was -0.24 which is largely consistent with an earlier meta-analysis ${ }^{30}$ that reported an effect size of -0.2 and together these data suggest a small but reliable association between eveningness and depression. Contrary to the findings of Au and 


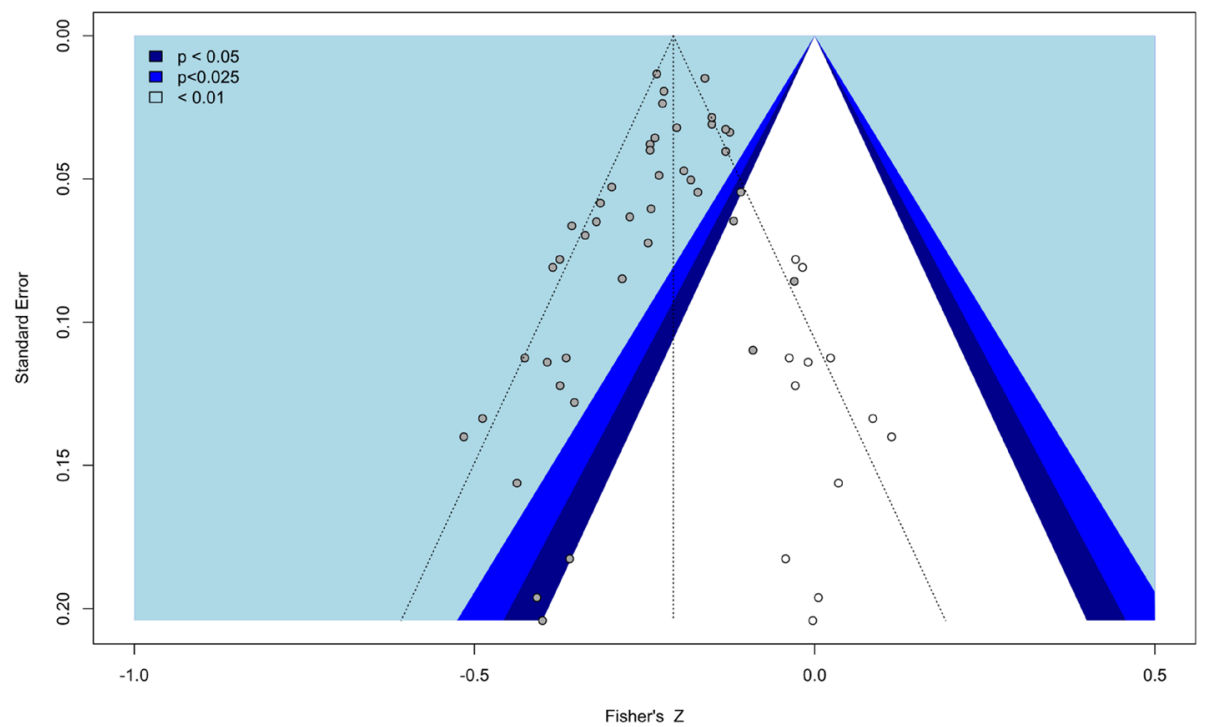

Figure 2. Contour funnel plot indicating potential risk of publication bias. Open dots represent the 12 studies imputed to achieve symmetry.

\begin{tabular}{|c|c|c|c|c|c|c|c|}
\hline \multirow{3}{*}{$\begin{array}{l}\text { Subgroup analyses } \\
\text { Sample composition }\end{array}$} & \multirow{2}{*}{\begin{tabular}{|l|} 
Description \\
Clinical
\end{tabular}} & \multirow{2}{*}{\begin{tabular}{|l|} 
Contributing effect sizes \\
7
\end{tabular}} & \multirow{2}{*}{$\begin{array}{l}\text { Fishers } Z \\
-0.27\end{array}$} & \multicolumn{2}{|l|}{ 95\% CI } & \multirow[t]{2}{*}{$Q$} & \multirow[t]{2}{*}{$p$ value } \\
\hline & & & & -0.37 & -0.17 & & \\
\hline & Non-clinical & 36 & -0.23 & -0.26 & -0.20 & 0.52 & 0.47 \\
\hline \multirow{2}{*}{ Publication year } & 2020 & 12 & -0.24 & -0.31 & -0.16 & & \\
\hline & Other year ${ }^{\&}$ & 31 & -0.24 & -0.27 & -0.21 & 0.00 & 0.94 \\
\hline \multirow{3}{*}{ Eveningness measure } & CSM & 11 & -0.24 & -0.31 & -0.17 & & \\
\hline & MEQ & 26 & -0.24 & -0.28 & -0.20 & & \\
\hline & rMEQ & 6 & -0.24 & -0.30 & -0.17 & 0.00 & 0.99 \\
\hline \multirow{3}{*}{ Depression measure } & BDI & 12 & -0.25 & -0.32 & -0.18 & & \\
\hline & CES-D & 10 & -0.23 & -0.28 & -0.18 & & \\
\hline & Other $^{\& \&}$ & 21 & -0.24 & -0.28 & -0.20 & 0.13 & 0.94 \\
\hline
\end{tabular}

Table 2. Subgroup analysis. Other year ${ }^{\&}=$ publication year other than 2020; Other ${ }^{\& \&}=$ any depression measure other than BDI or CES-D (see text for details). Q-test for between study heterogeneity, $p$ value for subgroup differences.

Reece, in the current analysis evidence of a potential publication bias (i.e. statistically significant or favourable results being more likely to be published than studies with non-significant or unfavourable results) was observed. The adjusted effect size (Fishers $Z=-0.21$ ), however, remained significant. Subgroup analyses demonstrated no moderating effect of sample characteristics, eveningness or depression measure, or studies published in 2020 vs. any other year. Meta-regression showed a significant effect of age on the association between eveningness and depression symptomatology, but no evidence for a moderating effect of sample size, gender ratio, or year of publication.

A long-standing question in the literature is one of directionality; does eveningness cause depression or is eveningness a consequence of the disorder? The cross-sectional studies quantified here cannot speak directly to this question. However, the current results demonstrated no significant difference between clinical and nonclinical samples, a finding consistent with $\mathrm{Au}$ and Reece ${ }^{30}$. Eveningness may therefore represent a risk-factor for depression rather than a consequence of the depressed state. The vulnerability-stress hypothesis of depression ${ }^{96,97}$ proposes that depression emerges through an interaction between psychological vulnerability factors (e.g., negative biases/preferential processing of negative material) and an environmental stressor (e.g., bereavement, financial insecurity). Importantly, previous work suggests that eveningness is associated with aspects of negative thinking (i.e. psychological vulnerability factors) in never-depressed individuals. For example, eveningness has been associated with greater recall for negative personality trait words, greater recognition of sad facial expressions ${ }^{63,98}$ and maladaptive emotion regulation strategies ${ }^{93,99}$. Similarly, high neuroticism (i.e. individuals who are emotionally reactive and tend to experience more negative emotions and depression) has also been associated with eveningness ${ }^{100}$. Converging evidence, therefore, suggests that in healthy, never-depressed individuals, eveningness is associated with depressogenic personality types, negative biases in emotional processing and impaired emotion 


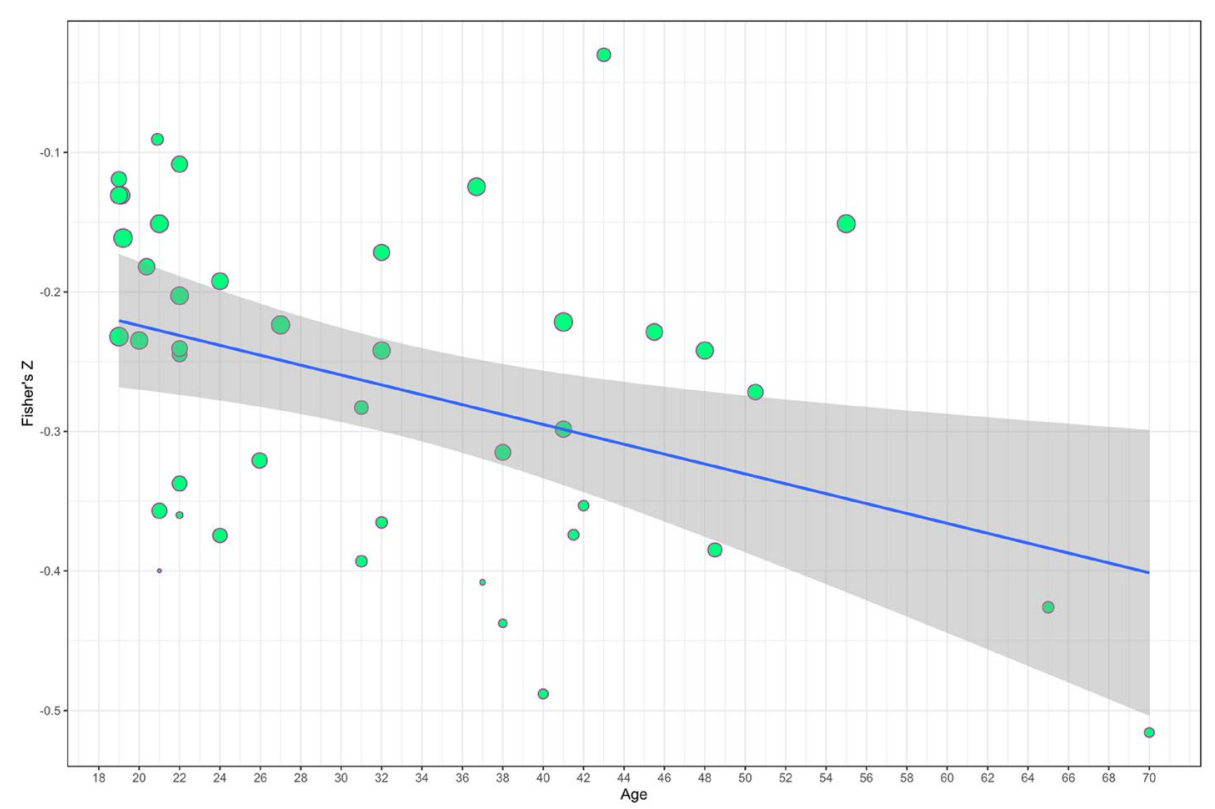

Figure 3. Regression of effect size (Fisher's $Z$ ) on age. Line of best fit plus confidence interval, bubbles show study weight.

regulation which, if combined with adversity, may lead to depression. These findings also suggest modifiable markers that could be therapeutically targeted to prevent the onset of depression in evening type individuals.

Of the moderators tested here only age was significantly associated with effect size. This contrasts with the findings of $\mathrm{Au}$ and Reece (2017) who did not observe a similar relationship. The mean age range in the current study was 19-70, which is broader than included by Au and Reece (19-55, MDD sample only) which may account for the discrepancy. Although it should be noted that for the majority of studies included here $(\sim 50 \%)$ the mean age was less than 30 years of age. Of note, Kim et al. recently reported no difference in prevalence rates for depression in late chronotypes $v s$. neither types in a population of Korean adults stratified by age (19-40, 41-59 and 60-80 years). However, although the total sample size was large $(N=6382)$ the number of participants in the older 60-80 years group classified as evening-type was small $(N=22)$ which may limit interoperability ${ }^{101}$. Counter to this, eveningness has been associated with increased odds for reporting depression in a large sample of older adults (age range 40-70 years) taken from the UK Biobank ${ }^{102}$. Similarly, here increasing age was associated with increased depressive symptomatology but the factors underpinning this effect remain to be elucidated. Older individuals that remain more evening-type may gradually lose friendship networks and group allegiances as peers gravitate to a social schedule in synchrony with their changing circadian typology, potentially leaving evening-prone individuals more isolated and potentially more prone to depression. This notion, however, is purely speculative and requires further investigation with suitably powered, prospective studies to determine the potential impact of age on the association between eveningness and depression.

There are several limitations associated with this work which should be considered when interpreting the results. A general limitation of meta-analyses is that by creating a summary of outcomes, important betweenstudy differences are ignored. To formally address this here study inclusion was restricted to adults, for clinical samples mood disorders other than MDD were excluded and only studies that used validated instruments to measure depressive symptomatology and diurnal preference were included. In addition, moderator analysis and meta-regression were employed to explore study heterogeneity. More specifically, the current analysis was unable to account for important factors that may impact the results. Sleep duration and/or sleep quality, for example, were not taking into consideration (zero-order correlations or unadjusted odds-ratios/mean differences were reported). Similarly, social jet-lag, the difference between internal rhythm and external demands (e.g. work or university), which may be more pronounced in evening-types and is associated with increased likelihood of reporting depressive symptoms ${ }^{103,104}$ was not included in this meta-analysis. The current report, therefore, cannot directly assess the potential impact of social jetlag on the association between eveningness and depressive symptoms. Further, the terms chronotype and diurnal preference are frequently used interchangeably in the literature but reflect different aspects of the same phenomenon. Here, the focus was diurnal preference and the questionnaires included limited to the MEQ, rMEQ and CSM which determine morningness/eveningness preferences based on self-reported preferences for times of activity and rest. These measures, therefore, reflect a personality trait. By contrast, instruments such as the Munich Chronotype Questionnaire (MCTQ) ${ }^{105}$ measure behaviour (mid-point of sleep on free days) which can be viewed as an indicator of state ${ }^{106}$. The focus of the current report was unipolar depression, but increasing evidence links eveningness with other affective disorders such as bipolar disorder ${ }^{107}$ and Major Depressive Disorder with Seasonal Pattern ${ }^{108}$ and anxiety ${ }^{109}$. Future metaanalyses that review and synthesise the recent literature related to these disorders is warranted. Finally, it should also be noted that all phases of this review and analyses were conducted solely by the author. 
In summary, the current meta-analysis demonstrated that eveningness is associated with depressive symptoms. These data are largely consistent with a previous meta-analysis $\mathrm{s}^{30}$ and the extant literature. The underlying causes that lead to depression are likely multifactorial and progress in understanding the links between diurnal preference and depression is predicated on a better understanding of the mechanistic underpinnings and suitably powered prospective studies that allow causal inference.

Received: 5 March 2021; Accepted: 24 May 2021

Published online: 07 June 2021

\section{References}

1. Logan, R. W. \& McClung, C. A. Rhythms of life: Circadian disruption and brain disorders across the lifespan. Nat. Rev. Neurosci. 20, 49-65 (2019).

2. Germain, A. \& Kupfer, D. J. Circadian rhythm disturbances in depression. Hum. Psychopharmacol. 23, 571-585 (2008).

3. Mendoza, J. Circadian insights into the biology of depression: Symptoms, treatments and animal models. Behav. Brain Res. 376, 112186 (2019).

4. Souêtre, E. et al. Circadian rhythms in depression and recovery: Evidence for blunted amplitude as the main chronobiological abnormality. Psychiatry Res. 28, 263-278 (1989).

5. Torquati, L., Mielke, G. I., Brown, W. J., Burton, N. W. \& Kolbe-Alexander, T. L. Shift work and poor mental health: A metaanalysis of longitudinal studies. Am. J. Public Health 109, e13-e20 (2019).

6. Katz, G., Knobler, H. Y., Laibel, Z., Strauss, Z. \& Durst, R. Time zone change and major psychiatric morbidity: The results of a 6-year study in Jerusalem. Comp. Psychiatry 43, 37-40 (2002).

7. Levandovski, R. et al. Depression scores associate with chronotype and social jetlag in a rural population. Chronobiol. Int. 28, 771-778 (2011).

8. Graw, P., Kräuchi, K., Wirz-Justice, A. \& Pöldinger, W. Diurnal variation of symptoms in seasonal affective disorder. Psychiatry Res. 37, 105-111 (1991).

9. Vadnie, C. A. \& McClung, C. A. Circadian rhythm disturbances in mood disorders: Insights into the role of the suprachiasmatic nucleus. Neural Plast. 2017, 1-28 (2017).

10. Ferrante, A. et al. Diurnal preference predicts phase differences in expression of human peripheral circadian clock genes. $J$. Circadian Rhythms 13, 5334 (2015).

11. Horne, J. A. \& Östberg, O. A self-assessment questionnaire to determine morningness-eveningness in human circadian rhythms. Int. J. Chronobiol. 4, 97-110 (1976).

12. Adan, A. \& Almirall, H. Horne \& Östberg morningness-eveningness questionnaire: A reduced scale. Personal. Individ. Differ. 12, 241-253 (1991).

13. Smith, C. S., Reilly, C. \& Midkiff, K. Evaluation of three circadian rhythm questionnaires with suggestions for an improved measure of morningness. J. Appl. Psychol. 74, 728-738 (1989).

14. Di Milia, L., Adan, A., Natale, V. \& Randler, C. Reviewing the psychometric properties of contemporary circadian typology measures. Chronobiol. Int. 30, 1261-1271 (2013).

15. Kantermann, T., Sung, H. \& Burgess, H. J. Comparing the morningnes--seveningness questionnaire and munich chronotype questionnaire to the dim light melatonin onset. J. Biol. Rhythms 30, 449-453 (2015).

16. Barclay, N. L., Watson, N. F., Buchwald, D. \& Goldberg, J. Moderation of genetic and environmental influences on diurnal preference by age in adult twins. Chronobiol. Int. 31, 222-231 (2014).

17. Barclay, N. L., Rowe, R., O'Leary, R., Bream, D. \& Gregory, A. M. Longitudinal stability of genetic and environmental influences on the association between diurnal preference and sleep quality in young adult twins and siblings. J. Biol. Rhythms 31, 375-386 (2016).

18. Fabbian, F. et al. Chronotype, gender and general health. Chronobiol. Int. 33, 864. https://doi.org/10.1080/07420528.2016.11769 27 (2016).

19. Kivelä, L., Papadopoulos, M. R. \& Antypa, N. Chronotype and psychiatric disorders. Curr. Sleep Med. Rep. https://doi.org/10. 1007/s40675-018-0113-8 (2018).

20. Taylor, B. J. \& Hasler, B. P. Chronotype and mental health: Recent advances. Curr. Psychiatry Rep. 20, 59 (2018).

21. Kitamura, S. et al. Evening preference is related to the incidence of depressive states independent of sleep-wake conditions. Chronobiol. Int. 27, 1797-1812 (2010).

22. Merikanto, I. et al. Evening types are prone to depression. Chronobiol. Int. 30, 719-725 (2013).

23. Hidalgo, M. P. et al. Relationship between depressive mood and chronotype in healthy subjects. Psychiatry Clin. Neurosci. 63, 283-290 (2009).

24. Antypa, N., Vogelzangs, N., Meesters, Y., Schoevers, R. \& Penninx, B. W. J. H. Chronotype associations with depression and anxiety disorders in a large cohort study. Depress. Anxiety https://doi.org/10.1002/da.22422 (2015).

25. Merikanto, I. et al. Circadian preference links to depression in general adult population. J. Affect. Disord. 188, 143-148 (2015).

26. Chan, J. W. Y. et al. Eveningness and insomnia: Independent Risk factors of nonremission in major depressive disorder. Sleep https://doi.org/10.5665/sleep.3658 (2014).

27. Gaspar-Barba, E. et al. Depressive symptomatology is influenced by chronotypes. J. Affect. Disord. 119, 100-106 (2009).

28. Bauducco, S., Richardson, C. \& Gradisar, M. Chronotype, circadian rhythms and mood. Curr. Opin. Psychol. 34, 77-83 (2020).

29. Taillard, J., Sagaspe, P., Philip, P. \& Bioulac, S. Sleep timing, chronotype and social jetlag: Impact on cognitive abilities and psychiatric disorders. Biochem. Pharmacol. 1, 114438. https://doi.org/10.1016/j.bcp.2021.114438 (2021).

30. Au, J. \& Reece, J. The relationship between chronotype and depressive symptoms: A meta-analysis. J. Affect. Disord. 218, 93-104 (2017).

31. Beck, A. T., Ward, C. H., Mendelson, M., Mock, J. \& Erbaugh, J. An inventory for measuring depression. Arch. Gen. Psychiatry 4, 561-571 (1961).

32. Zigmond, A. S. \& Snaith, R. P. The hospital anxiety and depression scale. Acta Psychiatr. Scand. 67, 361-370 (1983).

33. Hamilton, M. A rating scale for depression. J. Neurol. Neurosurg. Psychiatry 23, 56-62 (1960).

34. Lovibond, S. H. \& Lovibond, P. F. Manual for the Depression Anxiety Stress Scales (Psychology Foundation of Australa, 1995).

35. Brown, E. S. et al. The Quick Inventory of Depressive Symptomatology-Self-report: A psychometric evaluation in patients with asthma and major depressive disorder. Ann. Allergy Asthma Immunol. 100, 433-438 (2008).

36. Radloff, L. S. The CES-D scale a self-report depression scale for research in the general population. Appl. Psychol. Meas. 1, 385-401 (1977).

37. Zung, W. K. A self-rating depression scale. Arch. Gen. Psychiatry 12, 63-70 (1965).

38. Montgomery, S. A. \& Asberg, M. A new depression scale designed to be sensitive to change. Br. J. Psychiatry J. Ment. Sci. 134, 382-389 (1979). 
39. Kroenke, K., Spitzer, R. L. \& Williams, J. B. W. The PHQ-9. J. Gen. Intern. Med. 16, 606-613 (2001).

40. Kroenke, K., Spitzer, R. L., Williams, J. B. W. \& Löwe, B. An ultra-brief screening scale for anxiety and depression: The PHQ-4. Psychosomatics 50, 613-621 (2009).

41. Lee, M. B., Lee, Y. J., Yen, L. L., Lin, M. H. \& Lue, B. H. Reliability and validity of using a Brief psychiatric symptom rating scale in clinical practice. J. Formos. Med. Assoc. 89, 1081-1087 (1990).

42. Yesavage, J. A. et al. Development and validation of a geriatric depression screening scale: A preliminary report. J. Psychiatr. Res. 17, 37-49 (1982).

43. Roane, B. M. et al. Reliability of a scale assessing depressed mood in the context of sleep. TPM Test. Psychom. Methodol. Appl. Psychol. 20, 3-11 (2013).

44. Law, M. et al. Critical review form: Quantitative studies. J. Psychiatr. Res. 3, 1-10 (1995).

45. R Core Team. (R Foundation for Statistical Computing, 2020).

46. Makambi, K. H. The effect of the heterogeneity variance estimator on some tests of treatment efficacy. J. Biopharm. Stat. 14, 439-449 (2004).

47. Statistical Power Analysis for the Behavioral Sciences. (Erlbaum, 1988).

48. Cohen, J. Statistical Power Analysis for the Behavioral Sciences (Academic Press, 2013).

49. Bacaro, V. et al. Insomnia in the Italian population during covid-19 outbreak: A snapshot on one major risk factor for depression and anxiety. Front. Psychiatry 11, 579107 (2020).

50. Choi, H. J., Lee, Y. J., Yoo, Y. J., Cho, Y. W. \& Moon, H.-J. The effects of chronotype and social jetlag on medical students. Sleep Biol. Rhythms 17, 269-276 (2019).

51. Corruble, E. et al. Morningness: Eveningness and treatment response in major depressive disorder. Chronobiol. Int. 31, 283-289 (2014).

52. Hogben, A. L., Ellis, J., Archer, S. N. \& von Schantz, M. Conscientiousness is a predictor of diurnal preference. Chronobiol. Int. 24, 1249-1254 (2007).

53. Horne, C. M. \& Norbury, R. Late chronotype is associated with enhanced amygdala reactivity and reduced fronto-limbic functional connectivity to fearful versus happy facial expressions. Neuroimage 171, 355-363 (2018).

54. Hwang, J. Y., Kang, S.-G., Gwak, A. R., Park, J. \& Lee, Y. J. The associations of morningness-eveningness with anger and impulsivity in the general population. Chronobiol. Int. 33, 200-209 (2016).

55. Selvi, Y. et al. Associations between chronotype, sleep quality, suicidality, and depressive symptoms in patients with major depression and healthy controls. Chronobiol. Int. 27, 1813-1828 (2010).

56. Sultan, A., Taj, S., Choudhary, V. \& Parganiha, A. Predictive role of socio-demographic and chronotype on health-related quality of life of cancer patients from southeastern India. Biol. Rhythm Res. 1, 1-16 (2020).

57. Thapa, N. et al. The relationship between chronotype, physical activity and the estimated risk of dementia in community-dwelling older adults. Int. J. Environ. Res. Public. Health 17, 3701 (2020).

58. Üzer, A. \& Yücens, B. The effect of circadian preferences on insomnia severity and depressive symptoms via sleep hygiene in older adults with depression and healthy controls. Psychogeriatrics 20, 871-879 (2020).

59. Akram, U., Stevenson, J. C., Gardani, M., Akram, A. \& Allen, S. Psychopathy and chronotype disposition: The mediating role of depression. Heliyon 5, e2854 (2019).

60. Asarnow, L. D. et al. Circadian preference as a moderator of depression outcome following cognitive behavioral therapy for insomnia plus antidepressant medications: A report from the TRIAD study. J. Clin. Sleep Med. 15, 573-580 (2019).

61. Aydin, D., Selvi, Y., Kandeger, A. \& Boysan, M. The relationship of consumers' compulsive buying behavior with biological rhythm, impulsivity, and fear of missing out. Biol. Rhythm Res. 1, 1-9. https://doi.org/10.1080/09291016.2019.1654203 (2019).

62. Bakotic, M., Radosevic-Vidacek, B. \& Bjelajac, A. K. Morningness-eveningness and daytime functioning in university students: The mediating role of sleep characteristics. J. Sleep Res. 26, 210-218 (2017).

63. Berdynaj, D. et al. Effect of chronotype on emotional processing and risk taking. Chronobiol. Int. 1, 1-13. https://doi.org/10. 3109/07420528.2016.1146739 (2016).

64. Coleman, M. Y. \& Cain, S. W. Eveningness is associated with greater subjective cognitive impairment in individuals with selfreported symptoms of unipolar depression. J. Affect. Disord. 256, 404-415 (2019).

65. Furusawa, M. et al. Relationship between morningness-eveningness typology and cumulative fatigue or depression among Japanese male workers. Ind. Health 53, 361-367 (2015).

66. Haraszti, R. Á. et al. Morningness-eveningness interferes with perceived health, physical activity, diet and stress levels in working women: A cross-sectional study. Chronobiol. Int. 31, 829-837 (2014).

67. Hirata, F. C. et al. Depression in medical school: The Influence of morningness-eveningness. Chronobiol. Int. 24, 939-946 (2007).

68. Hou, T., Zhang, F., Mao, X. \& Deng, G. Chronotype and psychological distress among Chinese rural population: A moderated mediation model of sleep quality and age. PLoS ONE 15, 0241351 (2020).

69. Hsu, C.-Y., Gau, S.S.-F., Shang, C.-Y., Chiu, Y.-N. \& Lee, M.-B. Associations between chronotypes, psychopathology, and personality among incoming college students. Chronobiol. Int. 29, 491-501 (2012).

70. Inomata, Y., Echizenya, M., Takeshima, M., Shimizu, K. \& Shimizu, T. Validity and reliability of the Japanese version of the morningness-eveningness questionnaire evaluated from actigraphy. Sleep Biol. Rhythms 12, 289-296 (2014).

71. Jankowski, K. S. \& Dmitrzak-Weglarz, M. ARNTL, CLOCK and PER3 polymorphisms: Links with chronotype and affective dimensions. Chronobiol. Int. 34, 1105-1113 (2017).

72. Jankowski, K. S. Morningness-eveningness and depressive symptoms: Test on the components level with CES-D in Polish students. J. Affect. Disord. 196, 47-53 (2016).

73. Jeon, H. J., Bang, Y. R., Park, H. Y., Kim, S. A. \& Yoon, I.-Y. Differential effects of circadian typology on sleep-related symptoms, physical fatigue and psychological well-being in relation to resilience. Chronobiol. Int. 34, 677-686 (2017).

74. Kang, Y. et al. Testing the bidirectional associations of mobile phone addiction behaviors with mental distress, sleep disturbances, and sleep patterns: A one-year prospective study among Chinese College students. Front. Psychiatry 11, 10 (2020).

75. Khan, W. A. A., Conduit, R., Kennedy, G. A. \& Jackson, M. L. The relationship between shift-work, sleep, and mental health among paramedics in Australia. Sleep Health 6, 330-337 (2020).

76. Lau, E. Y. Y., Hui, C. H., Lam, J. \& Cheung, S.-F. Sleep and optimism: A longitudinal study of bidirectional causal relationship and its mediating and moderating variables in a Chinese student sample. Chronobiol. Int. 34, 360-372 (2017).

77. Lester, D. Morningness-eveningness, current depression, and past suicidality. Psychol. Rep. 116, 331-336 (2015).

78. Liberman, A. R., Halitjaha, L., Ay, A. \& Ingram, K. K. Modeling strengthens molecular link between circadian polymorphisms and major mood disorders. J. Biol. Rhythms 33, 318-336 (2018).

79. Lin, C.-Y. et al. Temporal associations between morningness/eveningness, problematic social media use, psychological distress and daytime sleepiness: Mediated roles of sleep quality and insomnia among young adults. J. Sleep Res. 1, e13076 (2020).

80. Markarian, S. A., Gildner, D. J., Pickett, S. M. \& Warnke, A. S. Morningness-eveningness and social anxiety symptoms: The influence of depression symptoms on the indirect effect through punishment sensitivity and experiential avoidance. Chronobiol. Int. 36, 214-224 (2019).

81. Müller, M. J., Olschinski, C., Kundermann, B. \& Cabanel, N. Patterns of self-reported depressive symptoms in relation to morningness-eveningness in inpatients with a depressive disorder. Psychiatry Res. 239, 163-168 (2016). 
82. Ong, J. C., Huang, J. S., Kuo, T. F. \& Manber, R. Characteristics of insomniacs with self-reported morning and evening chronotypes. J. Clin. Sleep Med. 3, 289-294 (2007).

83. Park, H., Lee, H.-K. \& Lee, K. Chronotype and suicide: The mediating effect of depressive symptoms. Psychiatry Res. 269, 316-320 (2018).

84. Park, Y.-M. Relationship between auditory evoked potentials and circadian preference in patients with major depressive episodes. Brain Sci. 10, 370 (2020).

85. Przepiorka, A., Blachnio, A. \& Cudo, A. Relationships between morningness, Big Five personality traits, and problematic Internet use in young adult university students: Mediating role of depression. Chronobiol. Int. 38, 248-259. https://doi.org/10.1080/07420 528.2020.1851703 (2020).

86. Randler, C., Stadler, L., Vollmer, C. \& Diaz-Morales, J. F. Relationship between depressive symptoms and sleep duration/chronotype in women. J. Individ. Differ. 33, 186-191 (2012).

87. Smagula, S. F. et al. Resting-state function connectivity associated with being a "morning-type" dementia caregiver and having lower depression symptom severity. J. Gerontol. 6, 115 (2020).

88. Sun, X., Gustat, J., Bertisch, S., Redline, S. \& Bazzano, L. The association between sleep chronotype and obesity among black and white participants of the Bogalusa Heart Study. Chronobiol. Int. 37, 123-134 (2020).

89. Togo, F., Yoshizaki, T. \& Komatsu, T. Association between depressive symptoms and morningness-eveningness, sleep duration and rotating shift work in Japanese nurses. Chronobiol. Int. 34, 349-359 (2017).

90. Toomey, R., Panizzon, M. S., Kremen, W. S., Franz, C. E. \& Lyons, M. J. A twin-study of genetic contributions to morningnesseveningness and depression. Chronobiol. Int. 32, 303-309 (2015).

91. Türkoğlu, G. \& Selvi, Y. The relationship between chronotype, sleep disturbance, severity of fibromyalgia, and quality of life in patients with fibromyalgia. Chronobiol. Int. 37, 68-81 (2020).

92. Üzer, A. \& Yücens, B. Chronotype and depressive symptoms in healthy subjects: The mediating role of hopelessness and subjective sleep quality. Chronobiol. Int. 37, 1173-1180 (2020).

93. Watts, A. L. \& Norbury, R. Reduced effective emotion regulation in night owls. J. Biol. Rhythms 32, 369-375 (2017).

94. Zhang, Y. et al. Caffeinated drinks intake, late chronotype, and increased body mass index among medical students in Chongqing, China: A multiple mediation model. Int. J. Environ. Res. Public. Health 15, 1-10 (2018).

95. Zhou, J. et al. Chronotype and depressive symptoms: A moderated mediation model of sleep quality and resilience in the 1styear college students. J. Clin. Psychol. 77, 340-355 (2021).

96. Hankin, B. L., Abramson, L. Y., Miller, N. \& Haeffel, G. J. Cognitive vulnerability-stress theories of depression: Examining affective specificity in the prediction of depression versus anxiety in three prospective studies. Cogn. Ther. Res. 28, 309-345 (2004).

97. Beck, A. T., Rush, A. J., Shaw, B. F. \& Emery, G. Cognitive Theory of Depression (Guildford, 1979).

98. Horne, C., Marr-Phillips, S. D. M., Jawaid, R., Gibson, E. L. \& Norbury, R. Negative emotional biases in late chronotypes. Biol. Rhythm Res. 48, 151-155 (2017).

99. Antypa, N. et al. Associations between chronotypes and psychological vulnerability factors of depression. Chronobiol. Int. 34, 1134. https://doi.org/10.1080/07420528.2017.1345932 (2017).

100. Duggan, K. A., Friedman, H. S., McDevitt, E. A. \& Mednick, S. C. Personality and healthy sleep: The importance of conscientiousness and neuroticism. PLoS ONE 9, e90628 (2014).

101. Kim, K. M., Han, S. M., Heo, K., Kim, W.-J. \& Chu, M. K. Sex differences in the association between chronotype and risk of depression. Sci. Rep. 10, 18512 (2020).

102. Norbury, R. Chronotype, depression and hippocampal volume: Cross-sectional associations from the UK Biobank. Chronobiol. Int. 36, 709-716 (2019).

103. Islam, Z. et al. Social jetlag is associated with an increased likelihood of having depressive symptoms among the Japanese working population: The Furukawa Nutrition and Health Study. Sleep 43, 10 (2020).

104. Wittmann, M., Dinich, J., Merrow, M. \& Roenneberg, T. Social jetlag: Misalignment of biological and social time. Chronobiol. Int. 23, 497-509 (2006).

105. Roenneberg, T., Wirz-Justice, A. \& Merrow, M. Life between clocks: daily temporal patterns of human chronotypes. J. Biol. Rhythms 18, 80-90 (2003).

106. Levandovski, R., Sasso, E. \& Hidalgo, M. P. Chronotype: a review of the advances, limits and applicability of the main instruments used in the literature to assess human phenotype. Trends Psychiatry Psychother. 35, 3-11 (2013).

107. Kanagarajan, K. et al. Morningness-eveningness questionnaire in bipolar disorder. Psychiatry Res. 262, 102-107 (2018).

108. Jankowski, K. S. Morningness-eveningness and seasonality. Biol. Rhythm Res. 48, 331-342 (2017).

109. Alvaro, P. K., Roberts, R. M. \& Harris, J. K. The independent relationships between insomnia, depression, subtypes of anxiety, and chronotype during adolescence. Sleep Med. 15, 934-941 (2014).

\section{Author contributions}

R.N. was responsible for reviewing the included studies, statistical analysis and manuscript preperation.

\section{Funding}

The author received no financial support for research or authorship. Funding to support publication fees was provided by Brunel University, London.

\section{Competing interests}

The author declares no competing interests.

\section{Additional information}

Supplementary Information The online version contains supplementary material available at https://doi.org/ 10.1038/s41598-021-91205-3.

Correspondence and requests for materials should be addressed to R.N.

Reprints and permissions information is available at www.nature.com/reprints.

Publisher's note Springer Nature remains neutral with regard to jurisdictional claims in published maps and institutional affiliations. 
(c) (i) Open Access This article is licensed under a Creative Commons Attribution 4.0 International cc) License, which permits use, sharing, adaptation, distribution and reproduction in any medium or format, as long as you give appropriate credit to the original author(s) and the source, provide a link to the Creative Commons licence, and indicate if changes were made. The images or other third party material in this article are included in the article's Creative Commons licence, unless indicated otherwise in a credit line to the material. If material is not included in the article's Creative Commons licence and your intended use is not permitted by statutory regulation or exceeds the permitted use, you will need to obtain permission directly from the copyright holder. To view a copy of this licence, visit http://creativecommons.org/licenses/by/4.0/.

(C) The Author(s) 2021 